\title{
SUMS AND PRODUCTS OF CYCLIC OPERATORS
}

\author{
PEI YUAN WU
}

(Communicated by Palle E. T. Jorgensen)

\begin{abstract}
It is proved that every bounded linear operator on a complex separable Hilbert space is the sum of two cyclic operators. For the product, we show that an operator $T$ is the product of finitely many cyclic operators if and only if the kernel of $T^{*}$ is finite-dimensional. More precisely, if $\operatorname{dim} \operatorname{ker} T^{*} \leq k$ $(2 \leq k<\infty)$, then $T$ is the product of at most $k+2$ cyclic operators. We conjecture that in this case at most $k$ cyclic operators would suffice and verify this for some special classes of operators.
\end{abstract}

A bounded linear operator $T$ on a complex Hilbert space $H$ is cyclic if there is a vector $x$ in $H$ such that $H$ is the closed linear span of the vectors $x, T x, T^{2} x, \ldots$ (in this case, $x$ is a cyclic vector of $T$ ). It is obvious that cyclic operators can act only on a separable space. Hence for the rest of the paper we will consider only Hilbert spaces which are separable.

For such a space $H$, let $\mathscr{C}(H)$ denote the set of all cyclic operators on $H$. Topological properties of this set have been studied quite extensively before. It was discovered that the size of $\mathscr{C}(H)$ relative to that of all operators $\mathscr{B}(H)$ depends very much on the dimension of the underlying space $H$. Thus if $H$ is finite-dimensional, then it is easy to show that $\mathscr{C}(H)$ is a dense open subset of $\mathscr{B}(H)$ (cf. [6, p. 499]). It follows in particular that the set of noncyclic operators is nowhere dense, whence $\mathscr{C}(H)$ is of the second category. However, for infinite-dimensional $H$, exactly the opposite is true. Indeed, it was proved in [5] that the set of noncyclic operators is dense in $\mathscr{B}(H)$. A refinement of that proof together with some Fredholm index theory, moreover, yields the existence of an open dense set of noncyclic operators (cf. [1, Proposition 11.18]). This implies that $\mathscr{E}(H)$ is nowhere dense, and thus noncyclic operators form a second category set in $\mathscr{B}(H)$.

In the following, we will show the abundance of cyclic operators in another sense. Indeed, in $\S 1$, after some preliminaries, we prove that every operator is the sum of two cyclic operators. This holds on any separable space regardless of its dimension. We then consider products of cyclic operators in $\S 2$. Our main theorem (Theorem 2.12 ) says that an operator $T$ is the product of finitely many cyclic operators if and only if $\operatorname{ker} T^{*}$ is finite-dimensional. To be more precise,

Received by the editors January 12, 1993 and, in revised form, March 2, 1993.

1991 Mathematics Subject Classification. Primary 47A05.

Key words and phrases. Cyclic operator, multicyclic operator, triangular operator.

This research was partially supported by the National Science Council of the Republic of China. 
we show that if $\operatorname{dim} \operatorname{ker} T^{*}=k \quad(2 \leq k<\infty)$, then $T$ is the product of at most $k+2$ cyclic operators. We suspect that the smallest number of cyclic operators required in this case should be $k$, which we pose as a conjecture. We are able to verify this for several classes of operators: operators on a finite-dimensional space, multicyclic operators, operators whose spectrum does not surround zero, isometries, coisometries, and normal operators.

\section{SuM}

We start with three propositions, which are the main tools that we use to prove our sum and product results. The first one reduces our consideration from the (additive or multiplicative) decomposition of general operators to that of cyclic operators. It appeared in [10, p. 463] and [4, Theorem 5].

Proposition 1.1. Every operator $T$ can be expressed in a triangular form

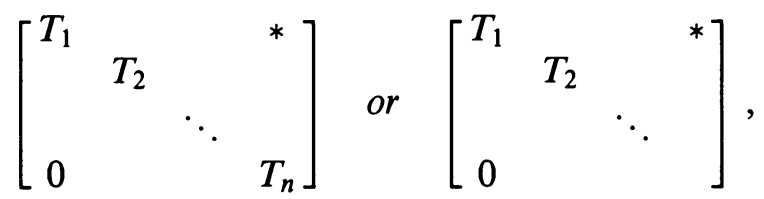

where $n$ is the multiplicity of $T$ and the $T_{j}$ 's are all cyclic.

Recall that the multiplicity of $T$ is the smallest cardinality of vectors $x_{1}, \ldots, x_{n}$ in $H$ for which $H$ is the closed linear span of the set $\left\{T^{k} x_{j}: k \geq 0\right.$ and $1 \leq j \leq n\}$. If $T$ is cyclic, then its multiplicity is 1 .

Our second proposition is quite well known (cf. [8, Problem 167]). It presents a special matrix form for every cyclic operator.

Proposition 1.2. An operator is cyclic if and only if it has the matrix representation

$$
\left[\begin{array}{cccc}
a_{1} & & & * \\
b_{1} & a_{2} & & \\
& b_{2} & \ddots & \\
& & \ddots & \\
0 & & &
\end{array}\right]
$$

with all $b_{n}$ 's nonzero.

Finally, we have

Proposition 1.3. If $T$ has the form

$$
\left[\begin{array}{llll}
T_{1} & & & * \\
& T_{2} & & \\
& & \ddots &
\end{array}\right]
$$

where the $T_{n}$ 's are all cyclic and have mutually disjoint spectra, then $T$ must be cyclic.

This result is an easy consequence of the following three facts: (1) if $T$ is of the above form, then $\sum_{n} \oplus T_{n}$ is a quasiaffine transform of $T$, that is, there is an injective operator $X$ with dense range such that $X\left(\sum_{n} \oplus T_{n}\right)=T X[3$, 
Proposition 2.5]; (2) if the $T_{n}$ 's are cyclic and have mutually disjoint spectra, then $\sum_{n} \bigoplus T_{n}$ is cyclic [11, Corollary 1.77]; (3) if $S$ is a quasiaffine transform of $T$ and $S$ is cyclic, then so is $T$.

The following corollary appeared in [9, Proposition 3.6].

Corollary 1.4. If

$$
T=\left[\begin{array}{lll}
a_{1} & & * \\
& a_{2} & \\
& & \ddots
\end{array}\right]
$$

is triangular with distinct $a_{n}$ 's, then the diagonal operator

$$
\operatorname{diag}\left(a_{n}\right)=\left[\begin{array}{ccc}
a_{1} & & 0 \\
& a_{2} & \\
& & \ddots \\
0 & &
\end{array}\right]
$$

is a quasiaffine transform of $T$ and $T$ is cyclic.

Now we are ready for our main result in this section.

Theorem 1.5. Every operator is the sum of two cyclic operators.

Proof. By Proposition 1.1, we may assume that

$$
T=\left[\begin{array}{cccc}
T_{1} & & & * \\
& T_{2} & & \\
& & \ddots &
\end{array}\right],
$$

where the $T_{n}$ 's are cyclic. (The proof for finitely many $T_{n}$ 's is the same.) Proposition 1.2 enables us to assume further that each $T_{n}$ is of the form

$$
\left[\begin{array}{cccc}
a_{n 1} & & & * \\
b_{n 1} & a_{n 2} & & \\
& b_{n 2} & \ddots & \\
0 & & \ddots &
\end{array}\right]
$$

with nonzero $b_{n j}$ 's. We have the decomposition

$$
\begin{aligned}
& {\left[\begin{array}{cccc}
a_{n 1} & & & * \\
b_{n 1} & a_{n 2} & & \\
& b_{n 2} & \ddots & \\
0 & & \ddots & \ddots
\end{array}\right]} \\
& =\left[\begin{array}{cccc}
c_{n 1}+d_{n} i & \bar{b}_{n 1} & & 0 \\
b_{n 1} & c_{n 2}+d_{n} i & \bar{b}_{n 2} & \\
& b_{n 2} & \ddots & \ddots \\
0 & & \ddots &
\end{array}\right]+\left[\begin{array}{ccc}
e_{n 1} & & \\
& e_{n 2} & \\
& & \ddots
\end{array}\right] \equiv S_{n}+R_{n},
\end{aligned}
$$


where the $c_{n j}$ 's and $d_{n}$ 's are all real, $d_{n}$ 's are distinct, and $e_{n j}$ 's are distinct. Hence

$$
T=\left[\begin{array}{llll}
S_{1} & & & * \\
& S_{2} & & \\
& & \ddots &
\end{array}\right]+\left[\begin{array}{llll}
R_{1} & & & 0 \\
& R_{2} & & \\
& & \ddots &
\end{array}\right] \equiv S+R
$$

In choosing the $c_{n j}$ 's and $d_{n}$ 's, we may assume that they are such that $\left\{\left\|S_{n}\right\|\right\}$ is bounded by a fixed number, say, $M$. Then $\left\|R_{n}\right\| \leq\left\|T_{n}\right\|+\left\|S_{n}\right\| \leq\|T\|+M$ for all $n$, whence $R$ is a bounded operator and therefore $S$ is a bounded operator. We now show that both $S$ and $R$ are cyclic. Since $S_{n}$ is the sum of the scalar $d_{n} i$ and a Hermitian operator, the distinctness of the $d_{n}$ 's implies that the spectra $\sigma\left(S_{n}\right)$ 's are mutually disjoint. Also, by Proposition 1.2, each $S_{n}$ is cyclic. Hence Proposition 1.3 implies that $S$ is cyclic. On the other hand, by Corollary 1.4, the diagonal operator $\operatorname{diag}\left(e_{n j}\right)$ is a quasiaffine transform of $R_{n}$. Hence $D=\sum_{n} \bigoplus \operatorname{diag}\left(e_{n j}\right)$ is a quasiaffine transform of $R=\sum_{n} \bigoplus R_{n}$. Since the $e_{n j}$ 's are all distinct, $D$ is a cyclic operator, whence $R$ is a cyclic operator. This completes the proof.

\section{Product}

In this section, we consider products of cyclic operators. An obvious necessary condition for an operator $T$ to be expressible as a product of $k \quad(1 \leq k<$ $\infty)$ cyclic operators is that $\operatorname{dim} \operatorname{ker} T^{*} \leq k$. Indeed, for $k=1$ this is trivial. Assuming its validity for $k$, we prove it for $k+1$. Let $T=T_{1} \cdots T_{k} T_{k+1}$ be a product of $k+1$ cyclic operators, and let $S=T_{1} \cdots T_{k}$. Then $T^{*}=T_{k+1}^{*} S^{*}$ implies that

$$
\begin{aligned}
\operatorname{dim} \operatorname{ker} T^{*} & =\operatorname{dim} \operatorname{ker} S^{*}+\operatorname{dim}\left(\operatorname{ran} S^{*} \cap \operatorname{ker} T_{k+1}^{*}\right) \\
& \leq \operatorname{dim} \operatorname{ker} S^{*}+\operatorname{dim} \operatorname{ker} T_{k+1}^{*} \leq k+1
\end{aligned}
$$

In view of the partial evidences discussed below, we suspect that, when $k \geq 2$, this necessary condition is also sufficient. This we propose as

Conjecture 2.1. An operator $T$ is the product of $k(2 \leq k<\infty)$ cyclic operators if and only if $\operatorname{dim} \operatorname{ker} T^{*} \leq k$.

We remark that in the above statement we may as well replace " $T$ is the product of $k$ cyclic operators" by " $T$ is the product of at most $k$ cyclic operators" due to the following lemma.

Lemma 2.2. Every cyclic operator is the product of two other cyclic operators.

Proof. Assume that

$$
T=\left[\begin{array}{cccc}
a_{1} & & & \\
b_{1} & a_{2} & \\
& b_{2} & \ddots & \\
0 & & \ddots &
\end{array}\right]
$$


where the $b_{n}$ 's are nonzero. We decompose

$$
T=\left[\begin{array}{llll}
c_{1} & & & 0 \\
& c_{2} & & \\
& & \ddots & \\
0 & & &
\end{array}\right]\left[\begin{array}{llll}
d_{1} & & & \\
e_{1} & d_{2} & \\
& e_{2} & \ddots & \\
0 & & \ddots &
\end{array}\right]
$$

where $c_{n}$ 's are distinct and the $e_{n}$ 's are all nonzero. Then $T$ is the product of two cyclic operators.

Falling short of proving the preceding conjecture in its full strength, we are only able to verify it for several classes of special operators. We start with the finite-dimensional case.

Proposition 2.3. On a finite-dimensional space, $T$ is the product of $k \quad(2 \leq k<$ $\infty)$ cyclic operators if and only if $\operatorname{dim} \operatorname{ker} T^{*} \leq k$.

Proof. We prove that $\operatorname{dim} \operatorname{ker} T^{*}=k$ implies that $T$ is the product of $k$ cyclic operators. Since the property of cyclicity is preserved under similarity, we may assume that $T$ is of the form

$$
\left[\begin{array}{cccc}
0 & 1 & & 0 \\
& 0 & \ddots & \\
& & \ddots & 1 \\
0 & & & 0
\end{array}\right] \oplus \cdots \oplus\left[\begin{array}{cccc}
0 & 1 & & 0 \\
& 0 & \ddots & \\
& & \ddots & 1 \\
0 & & & 0
\end{array}\right] \oplus\left[\begin{array}{ccc}
a_{1} & & * \\
& \ddots & \\
0 & & a_{n}
\end{array}\right] \equiv T_{1} \oplus \cdots \oplus T_{k} \oplus T_{k+1}
$$

where $T_{j}$ is of size $n_{j}$ for $j=1, \ldots, k+1$ and the $a_{i}$ 's are all nonzero. Note that

$$
T_{1} \oplus \cdots \oplus T_{k}=\left[\begin{array}{ccccc}
0 & 2 & & & 0 \\
& 0 & 3 & & \\
& & & \ddots & \\
& & & \ddots & N \\
0 & & & & 0
\end{array}\right]\left[\begin{array}{cccc}
b_{1} & & & 0 \\
& b_{2} & & \\
& & \ddots & \\
0 & & & b_{N}
\end{array}\right] \equiv S_{1} S_{2},
$$

where $N=n_{1}+\cdots+n_{k}$ and

$$
b_{j}= \begin{cases}1 / j & \text { if } 1 \leq j \leq N \text { and } \\ & j \neq n_{1}+1, n_{1}+n_{2}+1, \ldots, n_{1}+\cdots+n_{k-1}+1, \\ 0 & \text { otherwise }\end{cases}
$$

and that

$$
T_{k+1}=\left[\begin{array}{ccc}
b_{N+1} & & * \\
& \ddots & \\
0 & & b_{N+n}
\end{array}\right]\left[\begin{array}{ccc}
c_{1} & & 0 \\
& \ddots & \\
0 & & c_{n}
\end{array}\right] \equiv R_{1} R_{2},
$$

where $b_{N+1}, \ldots, b_{N+n}$ are all nonzero and distinct and the $c_{j}$ 's are nonzero and distinct and also distinct from the nonzero $b_{j}$ 's. Letting $A_{j}=S_{j} \oplus R_{j}$, $j=1,2$, we have $T=A_{1} A_{2}$. By Proposition $1.3, A_{1}$ is cyclic. On the other hand, since $A_{2}$ is a diagonal operator with $k-1$ zero diagonals, we can express it as a product of $k-1$ diagonal operators each with distinct diagonals. Hence $A_{2}$ is the product of $k-1$ cyclic operators, and therefore $T$ is the product of $k$ cyclic operators. 
Corollary 2.4. On an n-dimensional space, every operator is the product of $n$ cyclic operators and $n$ is the smallest such number.

Disposing of the finite-dimensional case, we move next to operators on infinite-dimensional spaces. Let the multiplicity of an operator $T$ be denoted by $m(T)$. Since $\operatorname{dim} \operatorname{ker} T^{*} \leq m(T)$ for any operator $T$, the next proposition is weaker than what we proposed in Conjecture 2.1. Recall that a multicyclic operator is one that has finite multiplicity.

Proposition 2.5. If $T$ is a multicyclic operator with multiplicity $m$, then $T$ is the product of $m$ cyclic operators.

Proof. We prove this by induction on $m$. Obviously, this is true for $m=1$. Assuming its validity for any operator $T$ with $m(T)=m$, we prove it for $m+1$. So let $T$ be an operator with multiplicity $m+1$. By [10, p. 463], we have the triangulation

$$
T=\left[\begin{array}{cc}
T_{1} & X \\
0 & T_{2}
\end{array}\right],
$$

where $T_{1}$ is cyclic and $m\left(T_{2}\right)=m$. Hence $T_{2}=S_{1} \cdots S_{m-1} S_{m}^{\prime}$ is a product of $m$ cyclic operators by the induction hypothesis. On the other hand, using Lemma 2.2 we obtain $T_{1}=R_{1} \cdots R_{m+1}$, where the $R_{j}$ 's are all cyclic and each $R_{j}, j=1, \ldots, m$, is diagonal and invertible with $\sigma\left(R_{j}\right)$ disjoint from $\sigma\left(S_{j}\right)$ when $j=1, \ldots, m-1$. Moreover, let $S_{m}^{\prime}=S_{m} S_{m+1}$, where both factors are cyclic, $\sigma\left(S_{m}\right)$ is disjoint from $\sigma\left(R_{m}\right)$ and $S_{m+1}$ is diagonal and invertible with $\sigma\left(S_{m+1}\right)$ disjoint from $\sigma\left(R_{m+1}\right)$. Finally, let

$$
Q_{j}=\left[\begin{array}{cc}
R_{j} & 0 \\
0 & S_{j}
\end{array}\right], \quad j=1, \ldots, m,
$$

and

$$
Q_{m+1}=\left[\begin{array}{cc}
R_{m+1} & R_{m}^{-1} \cdots R_{1}^{-1} X \\
0 & S_{m+1}
\end{array}\right] .
$$

Then each $Q_{j}$ is cyclic by Proposition 1.3 and $T=Q_{1} \cdots Q_{m+1}$.

To obtain other product results, we need the following lemma. It is an improvement over Lemma 2.2 for certain special cyclic operators. An operator is triangular if it can be represented in the matrix form

$$
\left[\begin{array}{lll}
a_{1} & & * \\
& a_{2} & \\
0 & & \ddots
\end{array}\right] .
$$

Lemma 2.6. If $T$ is a cyclic operator with dense range, then $T=T_{1} T_{2}$, where $T_{1}$ is unitary cyclic and $T_{2}$ is triangular cyclic.

Proof. By Proposition 1.2, we may assume that $T=\left[t_{i j}\right]$ with $t_{i, i-1} \neq 0$ for $i \geq 2$ and $t_{i j}=0$ for $i-j \geq 2$. In the following, we will construct a matrix $U=\left[u_{i j}\right]$ with the following properties:

(i) $U U^{*}=I$,

(ii) $u_{i, i+1} \neq 0$ for $i \geq 1$ and $u_{i j}=0$ for $j-i \geq 2$, and

(iii) $U T=\left[a_{i j}\right]$ with $a_{i j}=0$ for $i-j \geq 1$. 
This is done by first letting

$$
v_{1}=t_{21}
$$

and

$$
v_{n}=-\left(v_{1} t_{1, n-1}+\cdots+v_{n-1} t_{n-1, n-1}\right) / t_{n, n-1} \quad \text { for } n \geq 2 .
$$

Then, for each $n \geq 1$, let

$$
\alpha_{n}=\left|v_{n+1}\right| /\left(\sum_{j=1}^{n}\left|v_{j}\right|^{2}\right)^{1 / 2}\left(\sum_{j=1}^{n+1}\left|v_{j}\right|^{2}\right)^{1 / 2},
$$

$$
w_{n}= \begin{cases}-\left(\alpha_{n} / \bar{v}_{n+1}\right) \sum_{j=1}^{n}\left|v_{j}\right|^{2} & \text { if } v_{n+1} \neq 0, \\ 1 & \text { otherwise }\end{cases}
$$

and let $u_{n}=\left[u_{n 1} u_{n 2} \cdots\right]$, the $n$th row of $U$, be

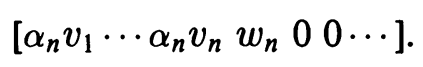

To verify (i), note that

$$
\begin{aligned}
\left\|u_{n}\right\|^{2} & =\left|\alpha_{n}\right|^{2} \sum_{j=1}^{n}\left|v_{j}\right|^{2}+\left|w_{n}\right|^{2} \\
& = \begin{cases}\left|v_{n+1}\right|^{2} /\left(\sum_{j=1}^{n+1}\left|v_{j}\right|^{2}\right)+\left(\sum_{j=1}^{n}\left|v_{j}\right|^{2}\right) /\left(\sum_{j=1}^{n+1}\left|v_{j}\right|^{2}\right)=1 & \text { if } v_{n+1} \neq 0, \\
0+1=1 & \text { otherwise }\end{cases}
\end{aligned}
$$

and, for $n>m \geq 1$, that

$$
\begin{aligned}
u_{n} \cdot u_{m} & =\sum_{j=1}^{m} \alpha_{n} v_{j} \bar{\alpha}_{m} \bar{v}_{j}+\alpha_{n} v_{m+1} \bar{w}_{m} \\
& =\alpha_{n}\left(\alpha_{m} \sum_{j=1}^{m}\left|v_{j}\right|^{2}+v_{m+1} \bar{w}_{m}\right)=0
\end{aligned}
$$

by (3)-(5). As for (ii), we have

$$
\begin{aligned}
\left|u_{n, n+1}\right| & =\left|w_{n}\right|=\left(\left|\alpha_{n}\right| /\left|v_{n+1}\right|\right) \sum_{j=1}^{n}\left|v_{j}\right|^{2} \\
& =\left(\sum_{j=1}^{n}\left|v_{j}\right|^{2}\right)^{1 / 2} /\left(\sum_{j=1}^{n+1}\left|v_{j}\right|^{2}\right)^{1 / 2} \geq\left|t_{21}\right| /\left(\sum_{j=1}^{n+1}\left|v_{j}\right|^{2}\right)^{1 / 2}>0
\end{aligned}
$$

if $v_{n+1} \neq 0$ by (1) and (3)-(5) and $u_{i j}=0$ for $j-i \geq 2$ by (5). Finally, for $i-j \geq 1$,

$$
a_{i j}=\sum_{k=1}^{j+1} u_{i k} t_{k j}=\alpha_{i} \sum_{k=1}^{j+1} v_{k} t_{k j}=0
$$

by (2) and (5), which verifies (iii). 
We next show that $U$ is actually unitary. In view of (i) above, we need only prove that $U$ is one-to-one. So let $x=\left[x_{1} x_{2} \cdots\right]^{\mathrm{t}}$ be such that $U x=0$. We will prove that $x$ also satisfies $T^{*} x=0$. Since $T$ has dense range, this would imply that $x=0$ as desired. We let $y=\left[y_{1} y_{2} \cdots\right]^{t}=T^{*} x$. Our goal can be accomplished by showing that (a) there are infinitely many nonzero $\alpha_{n}$ 's and (b) if $\alpha_{n} \neq 0$, then $y_{1}=\cdots=y_{n-1}=0$.

To prove (a), assume that there is some $n_{0} \geq 1$ such that $\alpha_{n}=0$ for all $n \geq n_{0}$. From (3), we have $v_{n+1}=0$ for $n \geq n_{0}$; hence, $v=\left[\bar{v}_{1} \bar{v}_{2} \cdots\right]^{\mathrm{t}}$ is a square-summable vector which satisfies $T^{*} v=0$ by (2). Since $T$ has dense range, this implies that $v=0$, whence $t_{21}=v_{1}=0$ by (1) contradicting our assumption. To prove (b), we assume that $\alpha_{n} \neq 0$ for some $n \geq 2$. From $U x=$ 0 , we deduce that $\left[x_{1} \cdots x_{n}\right]$ is orthogonal to $\left[\bar{u}_{i 1} \cdots \bar{u}_{i n}\right]$ for $i=1, \ldots, n-1$. These latter vectors together with $\left[\bar{u}_{n 1} \cdots \bar{u}_{n n}\right]$ are mutually orthogonal by (i). Since $\alpha_{n} \neq 0$ and $v_{1}=t_{21} \neq 0,\left[\bar{u}_{n 1} \cdots \bar{u}_{n n}\right]=\left[\alpha_{n} \bar{v}_{1} \cdots \alpha_{n} \bar{v}_{n}\right]$ is a nonzero vector. Hence $\left[x_{1} \cdots x_{n}\right]$ is a multiple of $\left[\bar{u}_{n 1} \cdots \bar{u}_{n n}\right]$. Since this latter vector is orthogonal to $\left[t_{1 j} \cdots t_{n j}\right]$ for all $j=1, \ldots, n-1$ by (iii), the same is true for $\left[x_{1} \cdots x_{n}\right]$. Thus

$$
y_{j}=\bar{t}_{1 j} x_{1}+\cdots+\bar{t}_{n j} x_{n}=0, \quad j=1, \ldots, n-1 .
$$

Let $A=U T$. Since $T=U^{*} A$ and $t_{i, i-1} \neq 0$ for all $i$, we deduce that the diagonals of $A$ are all nonzero. Hence it is possible to find a unitary diagonal operator $D$ such that the diagonals of the triangular operator $D A$ are all distinct. If $T_{1}=U^{*} D^{*}$ and $T_{2}=D A$, then $T=T_{1} T_{2}$ is the asserted factorization.

Now it is time to claim our rewards after such a laborious work. Note that if Conjecture 2.1 is indeed true, then every invertible operator should be the product of two cyclic operators. Not able to prove this, we show that the assertion is true under a more restricted condition. We say that a compact subset $K$ of $\mathbb{C}$ does not surround 0 if 0 is in the unbounded component of $\mathbb{C} \backslash K$ or, equivalently, if 0 is not in the polynomially convex hull of $K$.

Proposition 2.7. If the spectrum of $T$ does not surround 0 , then $T$ is the product of two cyclic operators.

Proof. By Proposition 1.1, we assume that $T$ is of the form $\left[T_{i j}\right]$, where $T_{i i}$ is cyclic for all $i$ and $T_{i j}=0$ for $i>j$. It is easily seen that $\sigma\left(T_{i i}\right)$ is contained in the polynomially convex hull of $\sigma(T)$ (cf. [12, Theorem 0.8]). Hence our hypothesis implies that $T_{i i}$ is invertible for all $i$. Thus Lemma 2.6 is applicable and we obtain $T_{i i}=U_{i} A_{i}$, where $U_{i}$ is unitary cyclic and $A_{i}$ is triangular cyclic. Let $\left\{r_{i}\right\}$ be a sequence of distinct real numbers between 1 and 2 , and let $V_{i}=r_{i} U_{i}$ and $B_{i}=A_{i} / r_{i}$. Then $T_{i i}=V_{i} B_{i}$. Since the $B_{i}$ 's together with the $A_{i}$ 's are all invertible, we may make further adjustments, as in the end of the proof of Lemma 2.6, so that the diagonals of all the $B_{i}$ 's are distinct. If

$$
T_{1}=\left[\begin{array}{cccc}
V_{1} & T_{12} B_{2}^{-1} & T_{13} B_{3}^{-1} & \\
& V_{2} & T_{23} B_{3}^{-1} & \ddots \\
& & V_{3} & \ddots \\
0 & & & \ddots
\end{array}\right] \text { and } T_{2}=\left[\begin{array}{cccc}
B_{1} & & & 0 \\
& B_{2} & & \\
& & B_{3} & \\
& & & \ddots
\end{array}\right] \text {, }
$$


then $T=T_{1} T_{2}$. Note that the relation $B_{i}^{-1}=r_{i} A_{i}^{-1}=r_{i} T_{i i}^{-1} U_{i}$ implies that $\left\|B_{i}^{-1}\right\| \leq 2\left\|T^{-1}\right\|$ for all $i$, whence $T_{2}$ is invertible and therefore $T_{1}$ is indeed a bounded operator. Since the $V_{i}$ 's are cyclic and their spectra are mutually disjoint, Proposition 1.3 implies that $T_{1}$ is cyclic. On the other hand, if $D_{i}$ is the diagonal operator whose diagonals are exactly those of $B_{i}$, then $D_{i}$ is a quasiaffine transform of $B_{i}$ by Corollary 1.4. Hence $\sum_{i} \oplus D_{i}$ is a quasiaffine transform of $T_{2}$. Since $\sum_{i} \oplus D_{i}$ is itself a diagonal operator with distinct diagonals, it is cyclic, whence $T_{2}$ is cyclic. This completes the proof.

The next result is another application of Lemma 2.6.

Theorem 2.8. Let $T=\sum_{n} \oplus T_{n}$, where the $T_{n}$ 's are cyclic. If $k \geq 2$ and $T_{n}$ has dense range for all $n>k$, then $T$ is the product of $k$ cyclic operators.

Proof. Using Lemma 2.2, we may express each $T_{n}, n=1, \ldots, k$, as a product $T_{n}=T_{n 1} \cdots T_{n k}$, where $T_{n j}$ 's are all cyclic and each $T_{n j}, j \neq n$, is a diagonal operator with spectrum disjoint from the spectra of all the other $T_{i j}$ 's. For the remaining $T_{n}$ 's, we use Lemma 2.6 to obtain $T_{n}=U_{n} A_{n}$, where $U_{n}$ is unitary cyclic and $A_{n}$ is triangular cyclic. We further express these $T_{n}$ 's as a product $T_{n}=T_{n 1} \cdots T_{n k} \quad(n>k)$, where each $T_{n 1}$ is a distinct multiple of $U_{n}$ with spectrum disjoint from the spectra of $T_{11}, \ldots, T_{k 1}$, and each $T_{n j}$, $j=2, \ldots, k$, is either triangular cyclic or diagonal cyclic with the closure of its distinct diagonals disjoint from the spectra of $T_{1 j}, \ldots, T_{k j}$. Let $S_{j}=$ $\sum_{n} \oplus T_{n j}, j=1, \ldots, k$. Obviously, $T=S_{1} \cdots S_{k} . S_{1}$ is cyclic by the above construction and Proposition 1.3. To prove the cyclicity of the remaining $S_{j}$ 's, let $D_{n j} \quad(n>k$ and $j=2, \ldots, k)$ be the diagonal operator with diagonals exactly those of $T_{n j}$. Since

$$
T_{1 j} \oplus \cdots \oplus T_{k j} \oplus \sum_{n=k+1}^{\infty} \bigoplus D_{n j}, \quad j=2, \ldots, k,
$$

is cyclic and is a quasiaffine transform of $S_{j}$ again by the above construction and Proposition 1.3, we conclude that $S_{j}$ is cyclic as asserted.

There are several corollaries of the preceding theorem. By the spectral theorem and the Wold decomposition, every isometry can be expressed as the direct sum of simple unilateral shifts and cyclic unitary operators. Thus Theorem 2.8 is applicable and we obtain

Corollary 2.9. An isometry $T$ is the product of $k(2 \leq k<\infty)$ cyclic operators if and only if $\operatorname{dim} \operatorname{ker} T^{*} \leq k$.

In a similar fashion, every coisometry is the direct sum of some backward shift and cyclic unitary operators. Since the former summand is cyclic [8, Problem 160], Theorem 2.8 implies the following corollary.

Corollary 2.10. Every coisometry is the product of two cyclic operators.

We remark that part of Corollary 2.9 also follows from a result of Halmos [7, Theorem 2] that an isometry $T$ with $\operatorname{dim} \operatorname{ker} T^{*}=k$ is the product of $k$ simple unilateral shifts and that Corollary 2.10 follows from the result of Brown [2, Theorem 3] that every coisometry is the product of some backward shift and a simple unilateral shift. 
For a normal operator $T$ with $\operatorname{dim} \operatorname{ker} T^{*}=k$, we have, by the spectral theorem, the decomposition $T=\sum_{n=1}^{\infty} \bigoplus T_{n}$, where $T_{1}, \ldots, T_{k}$ are the zero operators on a one-dimensional space and every $T_{n}(n>k)$ is one-to-one with dense range. Hence we have

Corollary 2.11. A normal operator $T$ is the product of $k(2 \leq k<\infty)$ cyclic operators if and only if $\operatorname{dim} \operatorname{ker} T^{*} \leq k$.

Concluding this paper, our final result says that products of (finitely many) cyclic operators can be characterized by the condition that the dimension of $\operatorname{ker} T^{*}$ be finite. It is obtained by combining the preceding three corollaries.

Theorem 2.12. An operator $T$ with $\operatorname{dim} \operatorname{ker} T^{*} \leq k \quad(2 \leq k<\infty)$ is the product of at most $k+2$ cyclic operators.

Proof. If $\operatorname{dim} \operatorname{ker} T \leq \operatorname{dim} \operatorname{ker} T^{*}$, then the polar decomposition of $T$ yields $T=V P$, where $V$ is an isometry with $\operatorname{dim} \operatorname{ker} V^{*}=\operatorname{dim} \operatorname{ker} T^{*}-\operatorname{dim} \operatorname{ker} T$ and $P=\left(T^{*} T\right)^{1 / 2}$ satisfies $\operatorname{ker} P=\operatorname{ker} T$. Hence, by Corollaries 2.9 and 2.11, $V$ and $P$ are, respectively, the products of $m$ and $n$ cyclic operators, where $m=\max \left\{\operatorname{dim} \operatorname{ker} T^{*}-\operatorname{dim} \operatorname{ker} T, 2\right\}$ and $n=\max \{\operatorname{dim} \operatorname{ker} T, 2\}$. It follows that $T$ is the product of $k+2$ cyclic operators.

On the other hand, if $\operatorname{dim} \operatorname{ker} T>\operatorname{dim} \operatorname{ker} T^{*}$, then consider the decomposition $T=P V$, where $P=\left(T T^{*}\right)^{1 / 2}$ and $V$ is a coisometry. Since $\operatorname{dim} \operatorname{ker} P=\operatorname{dim} \operatorname{ker} T^{*} \leq k$, Corollary 2.11 implies that $P$ is the product of $k$ cyclic operators. Also, $V$ is the product of two cyclic operators by Corollary 2.10. Hence in this case our assertion also follows.

Note that in the preceding proof we actually showed that $T$ is the product of $k$ cyclic operators if $2 \leq \operatorname{dim} \operatorname{ker} T$ and $\operatorname{dim} \operatorname{ker} T+2 \leq \operatorname{dim} \operatorname{ker} T^{*} \leq k$ $(2 \leq k<\infty)$.

\section{ADDED IN PROOF}

Applying the Gram-Schmidt process to the column vectors of a matrix, we can obtain an infinite-dimensional $Q R$ decomposition: every operator with dense range is the product of a unitary operator and a triangular operator. The proof of Lemma 2.6 can then be considerably shortened by using such an argument. This observation results from a sharp remark made by Professor Tzon-Tzer Lu in a presentation of results contained herein.

\section{REFERENCES}

1. C. Apostol, L. A. Fialkow, D. A. Herrero, and D. Voiculescu, Approximation of Hilbert space operators. II, Pitman, Boston, MA, 1984.

2. L. G. Brown, Almost every proper isometry is a shift, Indiana Univ. Math. J. 23 (1973), 429-431.

3. K. R. Davidson and D. A. Herrero, The Jordan form of a bitriangular operator, J. Funct. Anal. 94 (1990), 27-73.

4. R. G. Douglas and C. Pearcy, A note on quasitriangular operators, Duke Math. J. 37 (1970), 177-188.

5. P. A. Fillmore, J. G. Stampfli, and J. P. Williams, On the essential numerical range, the essential spectrum and a problem of Halmos, Acta Sci. Math. (Szeged) 33 (1962), 179-192.

6. I. Gohberg, P. Lancaster, and L. Rodman, Invariant subspaces of matrices with applications, Wiley, New York, 1986. 
7. P. R. Halmos, Products of shifts, Duke Math. J. 39 (1972), 779-787.

8. __ A Hilbert space problem book, 2nd ed., Springer-Verlag, New York, 1982.

9. D. A. Herrero, D. R. Lason, and W. R. Wogen, Semitriangular operators, Houston J. Math. 17 (1991), 477-499.

10. D. A. Herrero and W. R. Wogen, On the multiplicity of $T \oplus T \oplus \cdots \oplus T$, Rocky Mountain J. Math. 20 (1990), 445-466.

11. N. K. Nikolskii, Multiplicity phenomenon. I. An introduction and maxi-formulas, Toeplitz Operators and Spectral Function Theory (N. K. Nikolskii, ed.), OT, vol. 42, BirkhäuserVerlag, Basel (1989), 9-57.

12. H. Radjavi and P. Rosenthal, Invariant subspaces, Springer-Verlag, New York, 1973.

Department of Applied Mathematics, National Chiao Tung University, Hsinchu, TaIWAN, RePublic OF China 